Students at Belfast are "encouraged to see the hospice as a centre with specialised staff ...." Are they also taken actually to see a hospice? And if such an establishment is not available what steps are taken to allow students to come into contact with terminally ill patients in general hospital wards? In my survey of newly qualified hospital residents (junior house officers) $30 \%$ of the doctors had never participated in the clinical management of a single dying patient before graduation. ${ }^{1}$ A three hour teaching session may provide good groundwork, but unless students are more actively exposed to the terminally ill in the wards and at home they will be as inept as doctors who know 10 causes of pulmonary oedema but have never put in an intravenous line and given frusemide to a patient in acute left ventricular failure in the middle of the night.

Further on it is surprising to read that of the nine tutors taking part in the teaching session four are clergymen; in the small group sessions the students all meet a cleric but only a quarter see an oncologist or a general practitioner. Perhaps this (multidenominational) religious bias is peculiar to Northern Ireland; and no doubt all four ministers have expertise in this subject. I am aware of the importance of spiritual matters in terminal care, but in an "integrated and interdisciplinary" course why is there no nurse and no social worker?

S AHMEDZAI

Centre for Respiratory Investigation, Royal Infirmary,

1 Ahmedzai S. Dying in hospital: the resident's viewpoint. Br Med $\mathcal{F} 1982 ; 285: 712-4$.

\section{Premature loss of bone in chronic anorexia nervosa}

SIR,-The suggestion of Dr George I Szmukler and others (5 January, p 26) that chronic starvation Western style (anorexia nervosa) might be associated with osteoporosis (pathologically low whole bone density) is very much what one might expect, since the organic matrix of the skeleton is available as a source of energy when the mass of fat and muscle have become severely depleted. It is true that during the young adult period consolidation of the skeleton occurs and maximum bone mass is achieved. Hence starved people would be expected to reach the involutional (postmenopausal) state with a lower than normal bone mass. The corollary of this is the apparently greater than normal bone stock observed in a population of people who have had relative hyperalimentation during their formative years. ${ }^{1}$ Most authorities, however, would not agree that skeletal consolidation stops at or before the age of 20 years, and large population studies show that maximum whole bone density is achieved at about the age of 35 years. $^{2}$ If chronic anorexia nervosa is associated with osteoporosis it is more likely to be the result of failure to achieve optimal skeletal consolidation rather than that of premature skeletal involution, although the latter could play a part.

It is perhaps more important to ask ourselves whether anorexia nervosa is an important risk factor for osteoporosis. The authors screened the case notes of 140 patients with anorexia nervosa but came up with quantitative skeletal measurements on only three of them. Furthermore, their inter- pretation of the results is open to serious doubt. While their iliac crest biopsy results irrefutably support a diagnosis of osteoporosis, with all the values being more than 2 SD below the mean (found in a young healthy population), the measurements derived from metacarpal morphometry were all within the normal range and only one patient (case 4) had a distal forearm measurement ( $<470 \mathrm{mg}$ / $\left.\mathrm{cm}^{2}\right)$ in the osteoporotic region of the normal range. Furthermore, the authors use the term metacarpal index inaccurately, since this term refers specifically to the calculated ratio of cortical thickness:metacarpal diameter as measured on the second metacarpal and not to the calculated ratio of cortical area:metacarpal total cross sectional area, which is what they appear to have calculated. ${ }^{3}$

The authors state that a mineralisation defect (osteomalacia) was not found, but they would have to explain the raised alkaline phosphatase values found in all three patients and the hypocalcaemia in case 4 . Their assertion that oestrogen deficiency is synonymous with amenorrhoea in patients with anorexia nervosa requires substantiation. Plasma luteinising hormone values are meaningless in this context, and it would be important to establish that plasma oestrogen concentration, urinary oestrogen excretion, and vaginal cytological features in such patients were in the postmenopausal range before implicating oestrogen deficiency as an important risk factor for lower than normal whole bone density in anorexia nervosa.

Essex County Hospital, Eolchester, $3 \mathrm{NB}$

J M Aitken

1 Matkovic V, Kostial K, Simonovic I, Buzina R, Brodarec Á, Nordin BEC. Bone status and fracture Bates in two regions of Yugoslavia. Am $\not{J}$ Clin
Nutr 1979;32:540-9.

2 Genant HK, Cann CE, Ettinger B, et al. Quantitative computed tomography for spinal mineral assessBEC, Parfitt AM, Peck WA, Riggs BL, eds. Osteoporosis. Copenhagen: Aalborg Stiftsbogtrykkeri, $1984: 65-72$. 3 Aitken $M$. Measurement of bone mass and turnover.
In: Osteoporosis in clinical practice. Bristol: John Wright, 1984:19-43.

${ }^{*}{ }^{*}$ The authors reply below.-ED, BMF.

SIR,-We thank Dr Aitken for his comments. The most important finding in our study was of unequivocal osteoporosis on bone biopsy in three of our subjects with no evidence of osteomalacia and vertebral fractures in four of our patients.

Was this due to premature arrest of bone consolidation, on the one hand, and/or bone loss, on the other? The age at which bone consolidation in the various parts of the skeleton is maximal is not established with certainty, but if it is 30 for vertebral bone, then the former component would assume greater importance. Two of our patients had a late onset of anorexia nervosa, one at 27 and the other at 40 years. It seems unlikely that an irreversible termination of bone consolidation alone could result in the degree of osteoporosis noted. Starvation in anorexia nervosa tends to be selective for carbohydrates and fats, with a relative sparing of protein, and we do not know its precise effect on bone.

There is a consistent pattern of published reports pointing to very low oestrogen values with low luteinising hormone and follicle stimulating hormone values-that is, hypogonadotrophic hypogonadism-in patients with anorexia nervosa at the low weights shown by our patients. ${ }^{1}$ Therefore, the role of this hormone, well established in osteoporosis in other circumstances, merits strong consideration.

The bone density estimations in our patients showed one result below and two others just above the 2 SD mark, and these results are quite consistent with osteoporotic fracture in our clinic. The metacarpal index (metacarpal cortical area:total metacarpal area) ${ }^{2}$ was used in this study and correlates highly with bone mineral and skin collagen content ( $M$ Brincat et al, paper at International Symposium on Osteoporosis, Copenhagen, June 1984). That this measure of peripheral cortical bone was in the normal range suggests that these women lose trabecular bone faster than cortical bone, possibly related to their low body weight and long periods of inactivity. ${ }^{3}$ The raised alkaline phosphatase value in three of our patients is more difficult to explain. The source was not characterised but it could be the liver or gut. ${ }^{4}$ Victor Parsons

King's College Hospital,

London SE59RS

Institute of Psychiatry,
London SE5 8AF

1 Bell ET, Harkness RA, Loraine JA, Russell GFM Hormone assay studies in patients with anorexia Hormone assay studies in patients with
nervosa. Acta Endocrinol $1966 ; 85: 673-83$.

2 Nordin BEC. Clinical significance and pathogenesis ordin BEC. Cisical significance and p.
of osteoporosis. Br Med $\mathcal{F} 1971 ; \mathrm{i}: 571-6$.

3 Rigotti NA, Nussbaum SR, Herzog DB, Neer RM Osteoporosis in women with

4 Nordgren L, von Scheele C. Hepatic and pancreatic dysfunction in anorexia nervosa: a report of two cases. Biol Psychiatry 1977;12:681-6.

\section{Natural progesterone and} antihypertensive action

SIR,-It is interesting to read two articles printed in the 5 January issue side by side (pp 13 and 17). At first sight these articles, "Natural progesterone and antihypertensive action" and "Overview of randomised trials of diuretics in pregnancy," might appear unrelated. They do, however, both concern hypertension. I wonder why Dr Collins and colleagues review the use of diuretics instead of natural progesterone. No one is born diuretic or $\beta$ blocker deficient but there is plenty of evidence that some women, including those with pre-eclampsia, become progesterone deficient.

Dr Katharina Dalton, who has probably studied and written on the uses and applications of progesterone more extensively than anyone else, makes several references in her recent book to the use of progesterone for the treatment of pre-eclampsia. ${ }^{1}$ She urges that further research should be done on this subject to confirm her own earlier findings. ${ }^{2} 3$ Dr Dalton maintains that the use of progesterone for hypertension/pre-eclampsia has fallen into disrepute because obstetricians, and others in medicine, do not comprehend the essential differences betweeen natural progesterone and the several progestogens. Among other effects that progestogens can produce is masculinisation of a female fetus. It is salutary to remember that comparatively recently general practitioners were recommended to use depot progestogens for threatened miscarriage.

Dr P B Rylance and his colleagues claim that progesterone has an antihypertensive 